\title{
WILEY-VCH
}

DOI: $10.1002 /$ adom.201701163

Article type: Communication

\section{Periodic Planar-Homeotropic Anchoring Realized by Photo-Alignment for Stabilization of Chiral Superstructures}

Inge Nys, Ke Chen, Jeroen Beeckman*, Kristiaan Neyts

Inge Nys, Ke Chen, Prof. Dr. Jeroen Beeckman, Prof. Dr. Kristiaan Neyts

Technologiepark Zwijnaarde 15, Ghent, 9052, Belgium

Department of Electronics and Information Systems, Ghent University

E-mail: jeroen.beeckman@ugent.be

Keywords: liquid crystals, photo-alignment, polar and azimuthal anchoring, cholesteric fingers

Self-organization of chiral superstructures in liquid crystal (LC) materials is a powerful tool in developing functional devices based on soft matter. The control and manipulation of the helical axis orientation in chiral nematic liquid crystals (CLCs) are currently attracting significant attention, but are hindered by limited control over the anchoring at the interfaces. The development of new alignment techniques with local control over polar and azimuthal anchoring can open up a wide range of possibilities. Here a new photo-alignment process based on a mixture of photo-sensitive azo compounds and homeotropic alignment materials is developed and demonstrated. The alignment of the LC director near the surface depends on both intensity and polarization of the UV illumination. By two-beam interference UV illumination, periodic stripes of planar and homeotropic anchoring are realized. In addition it is demonstrated that the stripes of planar and homeotropic anchoring stabilize and align cholesteric fingers in cells with thickness smaller than the pitch. The 2D director configuration in the cholesteric fingers is identified by matching the experimental observations with numerical simulations based on finite element Q-tensor calculations. 


\section{WILEY-VCH}

Nematic liquid crystals (NLCs) have unique electro-optic properties and in particular chiral nematic liquid crystals (CLCs) can be used in a variety of applications such as mirror-less lasing, tunable diffraction gratings, color filters, lenses, displays, etc. ${ }^{[1]}$ Their selforganization into supramolecular structures together with their responsiveness to external stimuli offers them unique functional properties. ${ }^{[2]}$ Being able to control the alignment of LCs and especially CLCs on a micrometer-scale is therefore extremely important, both from a fundamental and an application-oriented point of view.

In this work we report on a new photo-alignment technique which allows micrometer-scale periodic variations of the anchoring. This alignment technique can be used to study the alignment of long-pitch CLC structures, as will be discussed, but also offers interesting possibilities for the realization of diffraction gratings and for the stable alignment of a shortpitch uniform lying helix for lasing and display applications. ${ }^{[4]}$ The novel method that we introduce for spatially controlling the polar and azimuthal anchoring at an interface allows us to obtain periodic stripes with planar and homeotropic anchoring with two-beam UV interference. The method is based on a mixture of homeotropic alignment material and azobased photo-alignment material illuminated with a periodic pattern of linearly polarized UV light. To the best of our knowledge, this is the first demonstration of controlling azimuthal versus polar anchoring by a mask-less UV interference illumination method. In the past, reversible switching between uniform homeotropic and planar alignment was demonstrated by using cis-trans photoisomerization and patterned homeotropic and planar alignment was achieved by depositing self-assembled monolayers of molecules. ${ }^{[6]}$ Chen et al. also established a photo-alignment technique that makes use of a mixture of nanoparticles and azodyes in the LC. ${ }^{[7]}$ In order to stabilize a lying helix structure, different methods were used to obtain a similar periodic anchoring: a polyimide layer with planar alignment was coated with a chromium complex surfactant for homeotropic anchoring and patterned by hard-contact photolithography. ${ }^{[4 a]}$ Alternatively, a mixture with photoreactive cholesteric monomer was 


\section{WILEY-VCH}

spincoated on top of an alignment layer with large pretilt and photopolymerized. ${ }^{[4 b]}$ The photo-alignment technique that we propose here is mask-less, allows high resolution and is based on the deposition of a single layer and therefore excels in versatility and ease of realization compared to previously reported methods. We expect that a further optimization of the technique can lead to full control of the azimuthal and polar alignment properties on a micrometer scale. This is not only interesting for the aforementioned applications but has very promising applications in geometrical phase optics and can lead to improved control over the mechanical response of liquid crystal elastomers. ${ }^{[5]}$ Moreover, Qian et al. found that spatially varying alignment can lead to multiple-degenerate orientational states in the bulk and that the effective anchoring strength can be tuned by varying the alignment texturing ${ }^{[8]}$ Additionally, the rewritability of photo-alignment materials might allow an in-situ optical manipulation and reorientation of the LC structures. In combination with stimuli-responsive LCs this may lead to a wealth of new possibilities. Both light stimuli, thermal stimuli and electrical stimuli have proven to be very promising for different applications. ${ }^{[2]}$

CLC in bulk spontaneously forms periodic helical structures and the distance over which the molecular director rotates by $2 \pi$ is called the pitch $p$. In the past, a lot of attention was given to the analyses of long pitch cholesteric structures in frustrated cells (small $d / p$ ratio with $d$ the cell tickness) or in microchannels with homeotropic boundary conditions. ${ }^{[3]}$ The director configuration in these type of cells strongly depends on the dielectric and elastic properties of the used CLC material, on the frustration ratio $\rho=d / p$ and on the applied voltage. Four different kinds of cholesteric fingers (CFs) can be identified and also the translationally invariant configuration often occurs in thin cells with homeotropic boundaries. ${ }^{[3 c, 3 e, 3 f, 3 g]}$ Confinement-unwound CLC systems also offer an interesting playground for the formation and self-assembly of bubble domains, triple-twisted elastic quasi-particles dubbed torons and skyrmionic particles. ${ }^{[3 \mathrm{i}, 3 \mathrm{k}, 31,3 \mathrm{~m}, 3 \mathrm{n}, 30,3 \mathrm{p}]}$ The formation of all these different structures relies on the existence of a competition between anchoring forces at the surface and the chirality of the 


\section{WILEY-VCH}

material. So far, geometrically frustrated CFs, in-plane lying helix structures and particle-like structures were mainly studied in cells with uniform alignment (homeotropic, tilted or planar). We apply our photo-alignment technique, with micrometer-scale periodic variations of the anchoring, to stabilize new CLC superstructures.

To obtain an alignment pattern with periodic stripes of planar and homeotropic anchoring we started from a mixture of homeotropic alignment material (SE 4811, 10wt $\%$ ) and photoalignment material (PAAD 22, 90wt\%). By using this mixture of compounds, homeotropic or planar alignment can be obtained, depending on the properties of the UV illumination. We designed an interference setup with two p-polarized beams with identical amplitude $\mathrm{E}_{0}$, incident in the xz plane, with an angle $\theta_{\mathrm{UV}}$ with the normal, so that the resulting electric field at the substrates is periodically varying as a function of $x$, as illustrated in Figure 1. In the plane of the substrate $(\mathrm{z}=0)$, the $\mathrm{x}$ - and $\mathrm{z}$-components of the incident electric field are given by (with $\mathrm{k}=2 \pi / \lambda, \omega=\mathrm{k} \mathrm{c} / \mathrm{n}$ ):

$E_{x}=2 E_{0} \sin \left(k \sin \left(\theta_{U V}\right) x\right) \cos \left(\theta_{U V}\right) \cos (\omega t)$

$E_{\mathrm{Z}}=-2 \mathrm{E}_{0} \cos \left(\mathrm{k} \sin \left(\theta_{\mathrm{UV}}\right) \mathrm{x}\right) \sin \left(\theta_{\mathrm{UV}}\right) \cos (\omega \mathrm{t})$

Because $+\bar{E}$ and $-\bar{E}$ give rise to the same alignment, the period of the photo-alignment is $\lambda /\left(2 \sin \left(\theta_{\mathrm{UV}}\right)\right)$. In our experiment $\lambda=355 \mathrm{~nm}$ and $\theta_{\mathrm{UV}} \approx 1.2^{\circ}$, yielding a period of $\approx 9 \mu \mathrm{m}$. The small angle between the interfering beams leads to a small value of $E_{z}$, negligible with respect to $E_{x}$.

Experimentally we observe that in the absence of UV illumination, the mixture gives rise to homeotropic alignment while strong linearly polarized UV illumination gives rise to planar alignment. Evidence for this is given in the supporting information (Figure S2). If the material is illuminated with two interfering UV beams, we observe stripes with planar alignment, separated by stripes with homeotropic alignment (Figure 1). From polarization microscopy, we find that homeotropic alignment is preserved in region 1 where $\mathrm{E}_{\mathrm{x}}$ is small, 


\section{WILEY-VCH}

while the LC has obtained planar orientation in region 2 (Figure 1). By adding a dichroic dye to the $\mathrm{LC}$ mixture we verified that the $\mathrm{LC}$ alignment in the region with planar orientation is along the y-axis (Figure S1 in the supporting information). This corresponds to reorientation of the photo-alignment molecules along the y-axis under influence of the electric field of the UV light in region 2. Only in the regions in which the photo-alignment material is welloriented by the UV illumination, planar alignment is dominant over the influence of the homeotropic material. The above mentioned mixture is therefore well-suited to obtain a periodic pattern of planar and homeotropic anchoring. We did not investigate the molecular structure of our alignment layer in detail and did not experimentally study the anchoring strength. However, we expect that the anchoring strength is weak near the border (between planar and homeotropic) and becomes stronger further away from the border. The exact variation in the anchoring strength will depend on the intensity profile of the illumination pattern and on the characteristics of the alignment mixture. Moreover, surface gliding of the director at the interfaces can influence the alignment strength in the transition region between the homeotropic and planar areas. Figure S2 (in the supporting information) shows lines with planar anchoring and a width close to $1 \mu \mathrm{m}$ at the edge of the illuminated area, which indicates that the polar anchoring strength in the planar regions is quite high (it is namely high enough to overcome the elastic energy of a rapid variation in the director).

This kind of periodic alignment is interesting for diffractive applications and is expected to have a strong influence on the alignment of CLCs. It is well known that in frustrated cells of CLC, in which the substrates are treated with a homeotropic alignment layer, the confinement ratio $\rho=d / p$ is important: for $\rho<1$ the CLC unwinds into a homeotropic state, while CFs are formed for slightly larger $\rho$. We show that the periodic alignment technique described in this article, can stabilize CFs for smaller values of the confinement ratio. The presence of stripes with preferential planar orientation strongly reduces the elastic energy in the CFs and 


\section{WILEY-VCH}

determines their orientation in the plane of the substrate. In Figure $\mathbf{2}$ experimental results are given for our cells with $\rho \approx 1 / 4$. The results demonstrate that in the non-illuminated area the CLC is unwound and homeotropically aligned, while in the central area illuminated with the UV interference pattern, a periodic structure of CFs is formed. Although the structure contains some defects, it clearly demonstrates that the periodic alignment pattern helps to stabilize and align CFs in confined geometries.

The thin orange lines that show up here and there in Figure 2 correspond to the director configuration making a $\pi$ twist which we call the CF3p configuration (due to the similarity with CF3 in homeotropic cells), where $\mathrm{p}$ stands for the partial planar alignment at the interfaces. This type of CF can be easily recognized since the planar region appears black at $0^{\circ}$ and bright at $45^{\circ}$ between crossed polarizers. The majority of the CFs in the area with periodic stripes of planar and homeotropic alignment are of a different type, because they are broader and consist of two bright lines with a darker line in the center. From the polarizing microscopy images (with the polarizers along $\mathrm{x}$ and $\mathrm{y}$ ) it is clear that the director of the CLC does not remain in the yz plane (as defined in Figure 1) because bright lines are visible. In some regions of the CFs the director obtains an important $\mathrm{x}$-component and with the help of numerical simulations we determine the director configuration.

Finite element (FE) simulations based on Q-tensor theory are performed to find the equilibrium director configuration and Jones calculus is used to find the corresponding transmission. ${ }^{[9]}$ The Q-tensor, which is a symmetric, traceless tensor order parameter of rank two is used to represent the LC. Nematic distortions correspond to spatial variations in the eigenvectors and eigenvalues of Q. The Landau-de Gennes free energy density contains a thermotropic bulk term, an elastic distortion term, an electrostatic energy term and a contribution of the surface energy density. ${ }^{[10 \mathrm{~b}-10 \mathrm{~d}]}$ In our simulations the elastic and dielectric properties of $\mathrm{E} 7$ are used $\left(\mathrm{K}_{11}=11.1 \mathrm{pN}, \mathrm{K}_{22}=6.5 \mathrm{pN}, \mathrm{K}_{33}=17.1 \mathrm{pN}\right)$ together with some 


\section{WILEY-VCH}

characteristic values for the bulk thermotropic coefficients. Very few measurements of thermotropic coefficients are found in the literature so we use thermotropic coefficients based on the ones measured for $5 \mathrm{CB}$ at a reduced temperature of $-2^{\circ}$, giving rise to an equilibrium order parameter of $0.54{ }^{[10]}$ As is common in Q-tensor simulations, we use 100 times smaller values for the thermotropic coefficients to increase the natural length scale of variations in the order parameters and to obtain faster numerical convergence. ${ }^{[10 \mathrm{~b}-10 \mathrm{~d}]}$ More details of the simulation method can be found in the supporting information and in previous articles. ${ }^{[9,10 b-}$ 10d]

In cells with homeotropic alignment filled with CLC, four types of CFs have been reported and the director profile in these CFs has been simulated and experimentally verified with fluorescence confocal polarizing microscopy. ${ }^{[3 c, 3 \mathrm{~d}, 3 \mathrm{e}, 3 \mathrm{f}, 3 \mathrm{~g}, 3 \mathrm{i}, 31]}$ Our simulation results for the three most common types of $\mathrm{CFs}(\mathrm{CF} 1, \mathrm{CF} 2$ and $\mathrm{CF} 3$, according to the classification of Oswald et al.) are illustrated in Figure 3. ${ }^{[3 g]}$ For ease of comparison, we use the same cell thickness $(4 \mu \mathrm{m})$ and width of the unit cell $(8 \mu \mathrm{m})$ as in our experiments, but the pitch is reduced to $\mathrm{p}=3.5 \mu \mathrm{m}$ to obtain stable CFs in a cell with homeotropic alignment $(\rho>1)$. The director configuration as a function of $\mathrm{x}$ and $\mathrm{z}$ is shown together with the representation on the unit sphere $\mathrm{S}^{2}$, according to the definition by Lequeux et al. ${ }^{[3 \mathrm{a}]}$ In this representation, the end point of the director on the unit sphere is shown as a function of $\mathrm{x}$, for a few discrete values of z. Only lines in the lower half of the cell are represented because the director in the top half can be deduced from symmetry arguments. The CF1 can continuously form from the translationally invariant configuration and easily nucleate from the homeotropic unwound structure. It does not involve dislincations and the director roughly makes a $2 \pi$ twist around an axis that is tilted away from the cell normal. The CF2 also involves a $2 \pi$ twist but cannot spontaneously nucleate from the homeotropic state and two point defects are required, one at either end of the finger. The metastable CF3 contains two twist disclination lines of opposite 


\section{WILEY-VCH}

sign (near the top and bottom substrate) and is not frequently observed. The CF3 is easily recognizable since it only has a $\pi$ twist and is therefore thinner than the other fingers.

The initial director configurations that we used in the simulations for cells with stripes of planar and homeotropic anchoring are inspired by the CFs in homeotropic cells. The cell thickness is $4 \mu \mathrm{m}$, the CLC pitch is $16 \mu \mathrm{m}$, the lateral dimension of the simulation area is $8 \mu \mathrm{m}$ and the width of the planar alignment region is set to $4 \mu \mathrm{m}$. An abrupt transition from strong homeotropic to strong planar alignment is assumed. Starting from the CF3 configuration and using the periodic boundary conditions at the interface, we find the stable CF3p configuration shown in Figure 3d. Away from the interfaces, this configuration is similar to a CF3 in a cell with homeotropic alignment. Because of the periodic planar/homeotropic alignment, each $\pi$ disclination that is present in the CF3 is replaced by two $\pi / 2$ disclinations at the edges of the alignment regions. The simulation results in Figure 3 show that also the CF1 and CF2 in homeotropic cells can be transformed into equivalent structures in our cells with stripes of planar and homeotropic anchoring. Both structures do not contain any singular disclinations and we call them CF1p and CF2p, with $\mathrm{p}$ again referring to the partial planar alignment. The difference in the director pattern between the CF1p (Figure 3i) and the CF1 in homeotropic cells (Figure 3a) is mainly related to the difference in pitch. In homeotropic cells CFs are only formed when the pitch is small $(\rho>1)$, while in our cells with periodic alignment the pitch is larger (smaller $\rho$ ). For a larger confinement ratio $\rho$ the twist axis is tilted further from the substrate normal and the structure better resembles the CF1 (Figure S2 in supporting information). We did not experimentally study geometries with shorter pitch ( $\rho$ values close to 1) but, based on the simulations, we can expect that the director configuration will continuously adapt towards a more twisted structure. This study demonstrates that a periodically varying alignment layer can induce chiral structures in thin devices $(d<p)$ that would otherwise give rise to a spatially invariant director configuration. 


\section{WILEY-VCH}

The simulated transmission through the cell in Fig. 3 is an approximation, because it is based on the Jones calculus (one calculation for every $\mathrm{x}$-coordinate) and does not take into account the spectrum of the incandescent microscope lamp. When the microscope images are compared to simulated transmissions, it is clear that the majority of the observed textures corresponds to the CF1p director configuration. The middle of the finger appears pink at $45^{\circ}$ and rather dark at $0^{\circ}$ while the edges are typically yellowish or greenish. The image is symmetric with respect to the middle of the CF1p and the transmission between crossed polarizers is brighter at $45^{\circ}$ between crossed polarizers than at $0^{\circ}$. The brightness of the experimental images (Figure 2) is adjusted to make the textures visible but also experimentally the transmission is higher when the fingers are oriented at $45^{\circ}$.

In summary, a new liquid crystal photo-alignment technique for full spatial control of planar and homeotropic anchoring was presented. The technique relies on a competition between homeotropic and planar alignment materials which is controlled by UV illumination. We have shown that UV interference illumination of the two-component alignment layer produces periodic stripes of homeotropic and planar anchoring, which could previously only be obtained with complicated techniques. The periodic planar and homeotropic anchoring at the surface can be made visible by filling the cell with NLC, and can also lead to complex and interesting 2D alignment patterns when the cell is filled with CLC. The periodic alignment stabilizes the formation of CFs, which appear for confinement ratios $\rho=d / p$ far below the unwinding transition in homeotropic cells. This opens up new possibilities regarding optical properties, electro-optical behavior, tunability, etc.

With the help of FE Q-tensor simulations the director configuration in 2D for different geometries was determined. We demonstrate that in devices with periodic alignment, the director configurations are equivalent to the well-known CF1, CF2 and CF3 in homeotropic 


\section{WILEY-VCH}

cells. The CFs observed experimentally are in good agreement with the simulations for the CF1p structure.

Our work demonstrates that it is possible to use spatially varying UV illumination to realize any azimuthal direction of planar alignment (using linearly polarized UV light with sufficient intensity) or homeotropic alignment (for weak UV illumination). ). We focused on a simple illumination technique with two interfering beams to demonstrate the usefulness of our method but with the help of a direct write system, a digital spatial light polarization converter, a mask illumination technique or a more complicated interference setup, a wide variety of complex alignment patterns should be achievable. ${ }^{[11]}$ This may be a very useful technique to realize new geometric phase optical components and develop NLC or CLC display modes, as for example the lying helix mode. In combination with stimuli-responsive LCs this can lead to a wealth of new phenomena and applications.

\section{Experimental Section}

Materials and cell preparation: A mixture of homeotropic alignment material (10 wt $\%$, SE 4811, Nissan Chemical Industries) and photo-alignment material (90 wt\%, PAAD 22, Beam Co.) is used. PAAD 22 is an azo-based compound that, after illumination with linearly polarized UV light, tends to align the LC director perpendicularly to the polarization direction. The glass substrates (Delta Technologies, ITO coated float glass $1.1 \mathrm{~mm}$ ) were treated with UV-ozone (15 min at $90^{\circ} \mathrm{C}$, Novascan) and after stirring $(>12 \mathrm{~h})$ the mixture was spincoated at $2500 \mathrm{rpm}$ for $15 \mathrm{sec}$. After $3 \mathrm{~min}$ prebake $\left(90^{\circ}\right)$ on the heating stage the samples were baked in the oven $\left(1 \mathrm{~h}, 180^{\circ}\right)$. The substrates were glued together using a glue (NOA68) with spherical spacer balls $(4 \mu \mathrm{m})$ near the edges. A mixture of the NLC E7 and the chiral dopant BDH 1305 was used in the experiments, resulting in a CLC with $\mathrm{p} \approx 16 \mu \mathrm{m}$. 


\section{WILEY-VCH}

Illumination: The cells were illuminated with a UV interference pattern $\left(\lambda=355 \mathrm{~nm}, \theta_{\mathrm{UV}} \approx 1.2^{\circ}\right.$, period $=9 \mu \mathrm{m}$ ) during 2 min with $70 \mathrm{~mW}$ over an area of $\approx 0.4 \mathrm{~mm}^{2}$ before being filled with LC above the nematic/isotropic transition temperature.

\section{Supporting Information}

Supporting Information is available from the Wiley Online Library or from the author.

\section{Acknowledgements}

The authors would like to acknowledge funding by the Flemish Fund for Scientific Research (FWO) in the form of a PhD Fellowship grant for I. Nys.

Received: Revised: Published online:

[1] a) H. Coles, S. Morris, Nat. Photonics 2010, 4, 676; b) Z.-G. Zheng, Y. Li, H. K. Bisoyi, L. Wang, T. J. Bunning, Q. Li, Nature 2016, 531, 352; c) J. Kobashi, H. Yoshida, M. Ozaki, Nat. Photonics 2016, 10, 389; d) D. Zhao, H. He, X. Gu, L. Guo, K. S. Wong, J. W. Y. Lam, B. Z. Tang, Adv. Opt. Mater. 2016, 4, 534; e) J. Beeckman, K. Neyts, P.J.M. Vanbrabant, Opt. Eng. 2011, 50, 081202; f) Z. Liu, P.-Z. Sun, W.-Q. Yang, X.-Q. Wang, D. Shen, Y. Li, F. Ye, Y.-Q. Lu, G. Li, Q. Li, Adv. Mater. 2017, 29, 1703165.

[2] a) L. Zhang, L. Wang, U. S. Hiremath, H. K. Bisoyi, G. G. Nair, C. V. Yelamaggad, A. M. Urbas, T. J. Bunning, Q. Li, Adv. Mater. 2017, 29, 1700676; b) Z.-G. Zheng, R. S. Zola, H. K. Bisoyi, L. Wang, Y. Li, T. J. Bunning, Q. Li, Adv. Mater. 2017, 29, 1701903; c) H.-C. Jau, Y. Li, C.-C. Li, C.-W. Chen, C.-T. Wang, H. K. Bisoyi, T.-H. Lin, T. J. Bunning, Q. Li, Adv. Opt. Mater. 2015, 3, 166; d) H. K. Bisoyi, Q. Li, Chem. Rev. 2016, 116, 15089.

[3] a) F. Lequeux, P. Oswald, Phys. Rev. A 1989, 40, 3974; b) M. Kawachi, O. Kogure, Y. Kato, Jpn. J. Appl. Phys. 1974, 13, 1457 ; c) I. Smalyukh, B. Senyuk, P. Palffy-Muhoray, O. D. Lavrentovich, H. Huang, E. C. Gartland, V. H. Bodnar, T. Kosa, B. Taheri, Phys. Rev. E 2005, 72, 061707; d) J. Baudry, S. Pirkl, P. Oswald, Phys. Rev. E 1999, 59, 5562; e) J. Baudry, S. Pirkl, P. Oswald, Phys. Rev. E 1998, 57, 3038; f) L. Gil, J.M. Gilli, Phys. Rev. Lett. 1998, 


\section{WILEY-VCH}

80, 5742; g) P. Oswald, J. Baudry, S. Pirkl, Phys. Rep. 2000, 337, 67; h) I. Gvozdovskyy, O. Yaroshchuk, M. Serbina, R. Yamaguchi, Opt. Express 2012, 20, 3499; i) I. Smalyukh, D. Kaputa, A. V. Kachynski, A. N. Kuzmin, P. J. Ackerman, C. W. Twombly, T.Lee, R. P. Trivedi, P. N. Prasad, Opt. Express 2012, 20, 6870; j) O. Yaroshchuk, S. Tomylko, I.

Gvozdovskyy, R. Yamaguchi, Appl. Opt. 2013, 52, E53; k) P. J. Ackerman, J. V. D. Lagemaat, I. Smalyukh, Nat. Commun. 2015, 6, 6012; 1) I. Smalyukh, Y. Lansac, N. A. Clark, R. P. Trivedi, Nat. Mater. 2010, 9, 139; m) C. Loussert, E. Brasselet, Appl. Phys. Lett. 2014, 104, 5; n) C. Loussert, S. Iamsaard, N. Katsonis, E. Brasselet, Adv. Mater. 2014, 26, 4242; o) A. Varanytsia, L.-C. Chien, Opt. Lett. 2015, 40, 4392; p) A. Leonov, I. Dragunov, U. Robler, A. Bogdanov, Phys. Rev. E 2014, 90, 042502; q) P. Oswald, A. Dequidt, Phys. Rev. E 2008, 77, 051706; r) Y. Guo, S. Afghah, J. Xiang, O. D. Lavrentovich, R. L. B. Selinger, Q.-H. Wei, Soft Matter 2016, 12, 6312.

[4] a) L. Komitov, G. P. Bryan-Brown, E. L. Wood, A. B. J. Smout, J. Appl. Phys. 1999, 86, 3508; b) G. Hegde, L. Komitov, Appl. Phys. Lett. 2010, 96, 113503; c) G. Carbone, D.

Corbett, S. J. Elston, P. Raynes, A. Jesacher, R. Simmonds, M. Booth, Mol. Cryst. Liq. Cryst. 2011, 544, 37/[1025]; d) I. Nys, J. Beeckman, K. Neyts, in Proc. SPIE, Vol. 9565 (Eds: I. C. Khoo) San Diego, United States 2015, Liquid Crystals XIX.

[5] a) S. Slussarenko, A. Alberucci, C. P. Jisha, B. Piccirillo, E. Santamato, G. Assanto, L. Marrucci, Nat. Photonics 2016, 10, 571; b) T. H. Ware, M. E. McConney, J. J. Wie, V. P. Tondiglia, T. J. White, Science 2015, 347, 982; c) M. Hendrikx, A. P. H. J. Schenning, D. J. Broer, Soft Matter 2017, 13, 4321.

[6] a) V. K. Gupta, N. L. Abbott, Science 1997, 276, 1533; b) S. Park, C. Padeste, H. Schift, J. Gobrecht, T. Scharf, Adv. Mater. 2005, 17, 1398 ; c) G. Barbero, V. Popa-Nita, Phys. Rev. E 2000, 61, 6696 ; d) H. Nadasi, R. Stannarius, A. Eremin, A. Ito, K. Ishikawa, O. Haba, K. Yonetake, H. Takezoe, F. Araoka, Phys. Chem. Chem. Phys. 2017, 19, 7597.

[7] W.-Z. Chen, Y.-T. Tsai, T.-H. Lin, Appl. Phys. Lett. 2009, 94, 201114. 


\section{WILEY-VCH}

[8] T.-Z. Qian, P. Sheng, Phys. Rev. E 1997, 55, 7111.

[9] a) R. James, E. Willman, F. A. Fernández, S. E. Day, IEEE Trans. Electron Devices 2006, 53, 1575; b) I. Nys, J. Beeckman, K. Neyts, Soft Matter 2015, 11, 7802.

[10] a) H. J. Coles, Mol. Cryst. Liq. Cryst. 1978, 49, 67; b) L. A. Parry-Jones, R. B. Meyer, S. J. Elston, J. Appl. Phys. 2009, 106, 014510; c) E. Willman, F. A. Fernández, R. James, S. E. Day, J. Disp. Technol. 2008, 4, 276; d) N. J. Mottram, C. M. Care, D. J. Cleaver, Phys. Rev. E 2006, 74, 041703 .

[11] a) L. De Sio, D. E. Roberts, Z. Liao, S. Nersisyan, O. Uskova, L. Wickboldt, N. Tabiryan, D. M. Steeves, B. R. Kimball, Opt. Express 2016, 24, 18297; b) K. Kawai, M. Sakamoto, K. Noda, T. Sasaki, N. Kawatsuki, H. Ono, J. Appl. Phys. 2016, 119, 123102; c) M. N.

Miskiewicz, M. J. Escuti, Opt. Eng. 2015, 54, 025101. 


\section{WILEY-VCH}

Figure 1. a) UV interference illumination setup (CW = continuous wave, $\mathrm{HWP}=$ half-wave plate, $\mathrm{PBS}=$ polarizing beam splitter); b) Periodically varying polarization pattern at the substrate created by two-beam interference; c-f) Polarizing microscopy images of a cell filled with NLC for different magnifications and different orientations with respect to the polarizers. In c) the surface periodicity is aligned with the polarizer while in d-f) the surface periodicity is rotated $45^{\circ}$ with respect to the polarizers. A schematic representation of the director configuration is given in e) (dot: homeotropic alignment, line: planar alignment).

(a)

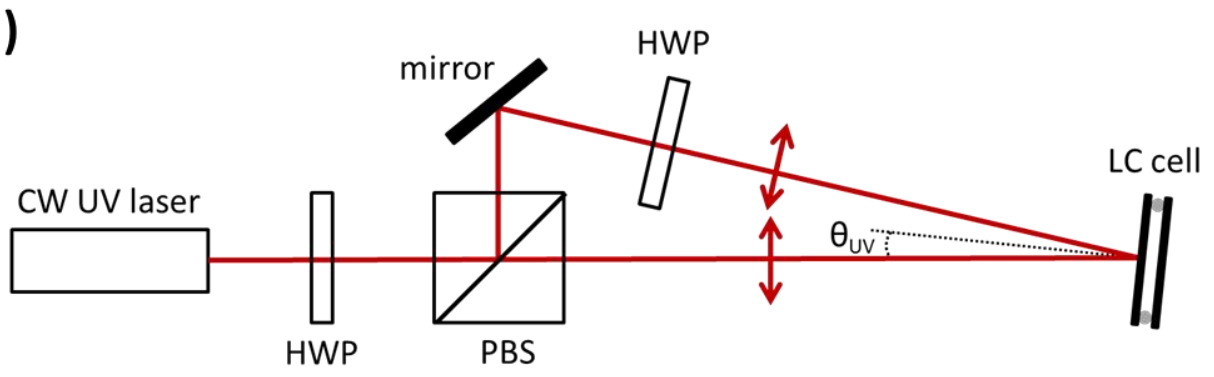

(b)

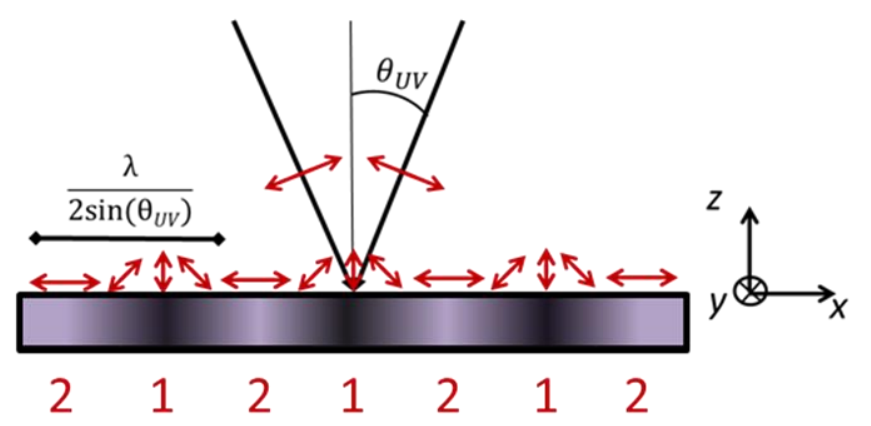

(c)

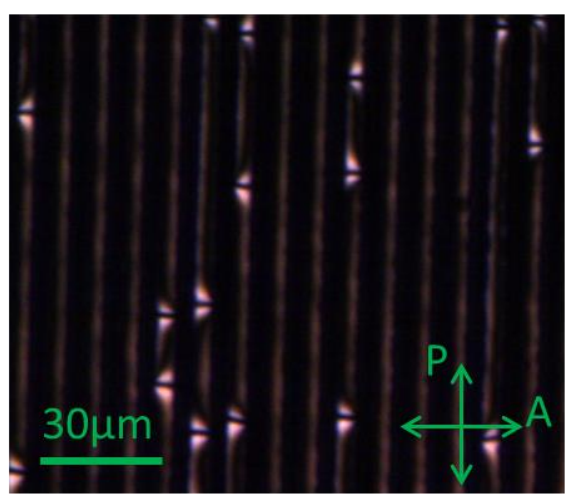

(e)

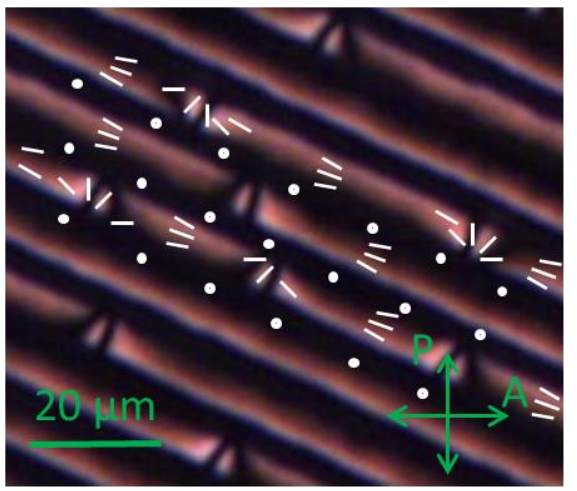

(d)

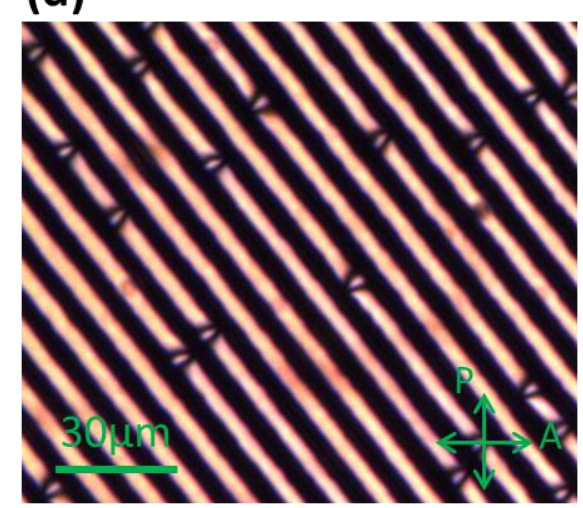

(f)

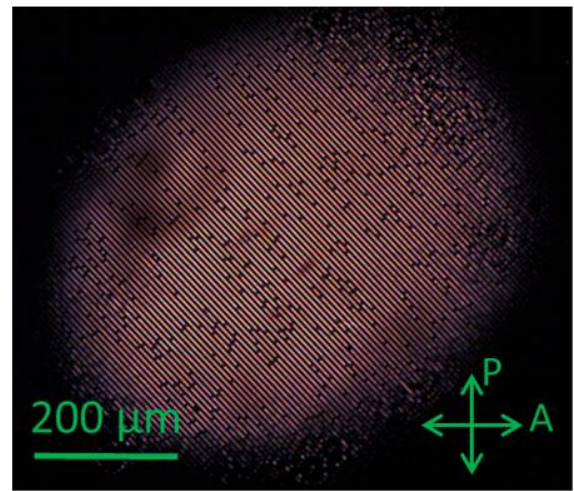




\section{WILEY-VCH}

Figure 2. Polarizing microscopy images of a cell filled with long pitch CLC ( $p=16 \mu \mathrm{m}$, $d=4 \mu \mathrm{m}$ ) with a smaller ( $\mathrm{a}$ and $\mathrm{b}$ ) and a larger (c-e) magnification. $\mathrm{a}-\mathrm{b}$ ) The illuminated area is surrounded by a homeotropic unwound region. c-e) in the illuminated area mainly CF1p structures are observed. Figures a and $\mathrm{c}$ are taken shortly after filling the cell, the others (b,d,e) after two months.

(a)

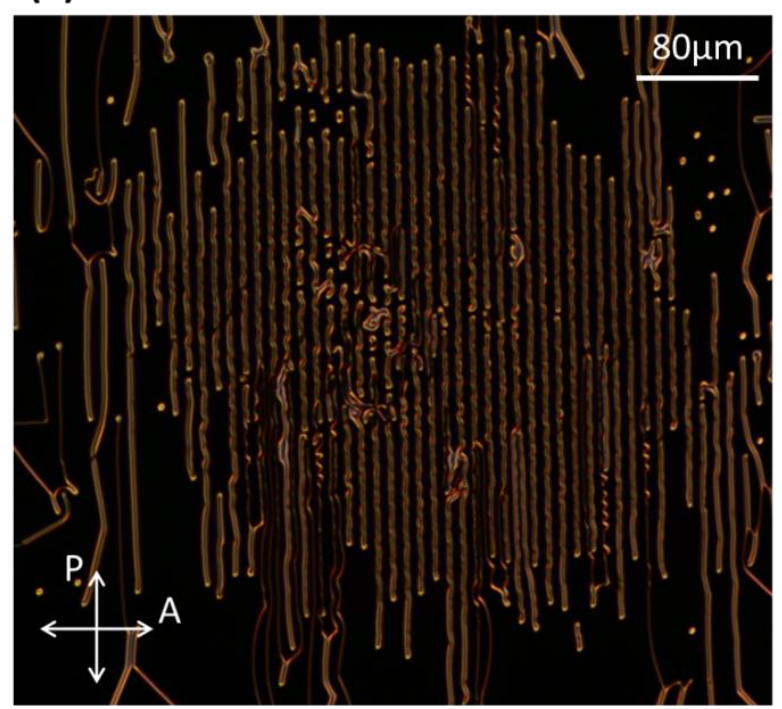

(c)

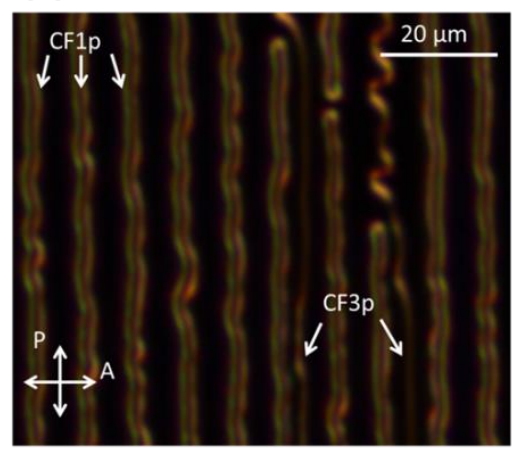

(b)

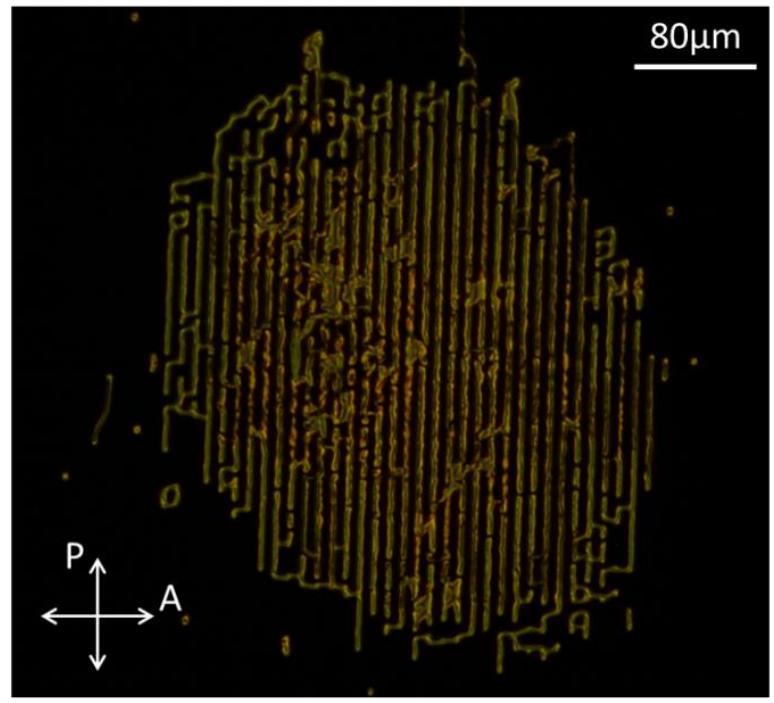

(e)
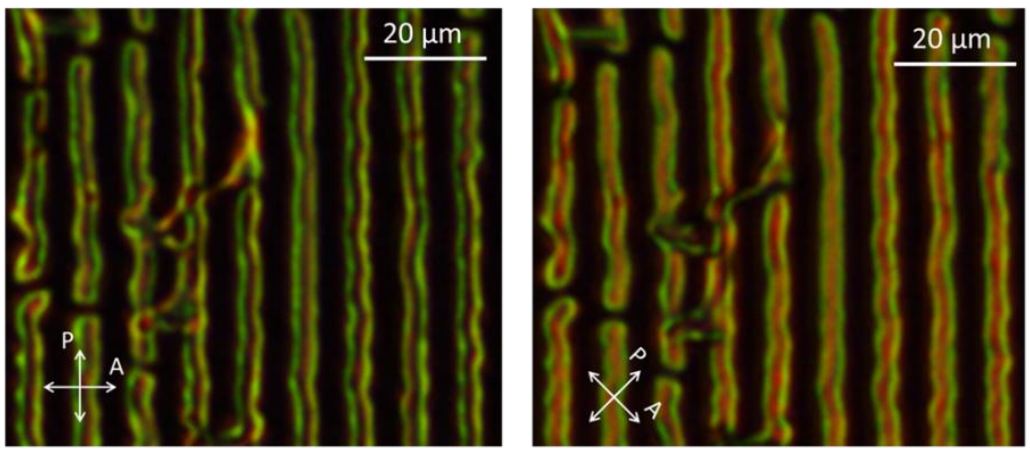


\section{WILEY-VCH}

Figure 3. a-c) simulated director configuration and representation on the unit sphere for CF1 (a), CF2 (b) and CF3(c) in a cell with homeotropic alignment ( $d=4 \mu \mathrm{m}, p=3.5 \mu \mathrm{m})$. i-iii) Simulated director configuration, representation on the unit sphere and transmission between crossed polarizers for CF1p (i), CF2p (ii) and CF3p (iii) in a cell with periodic stripes of planar and homeotropic alignment $(d=4 \mu \mathrm{m}, p=16 \mu \mathrm{m})$. A cross section (xz-plane) perpendicular to the finger axis and the representation on the unit sphere are shown. The perpendicular transmission is simulated as a function of $\mathrm{x}$.

(a)
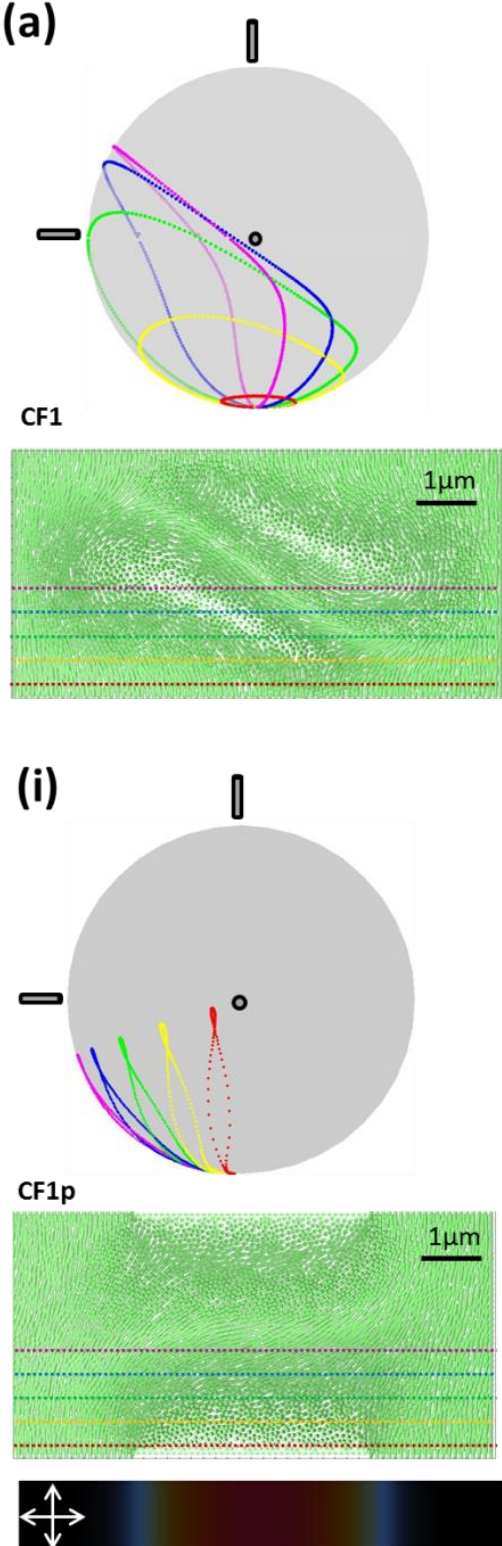

$\pi$ (b)

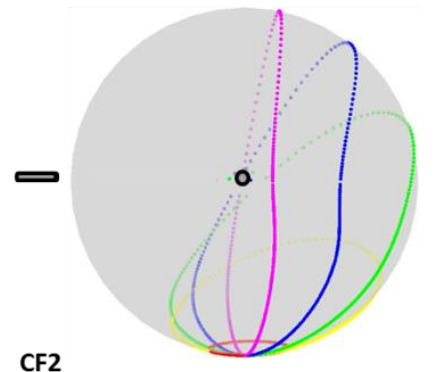

CF2
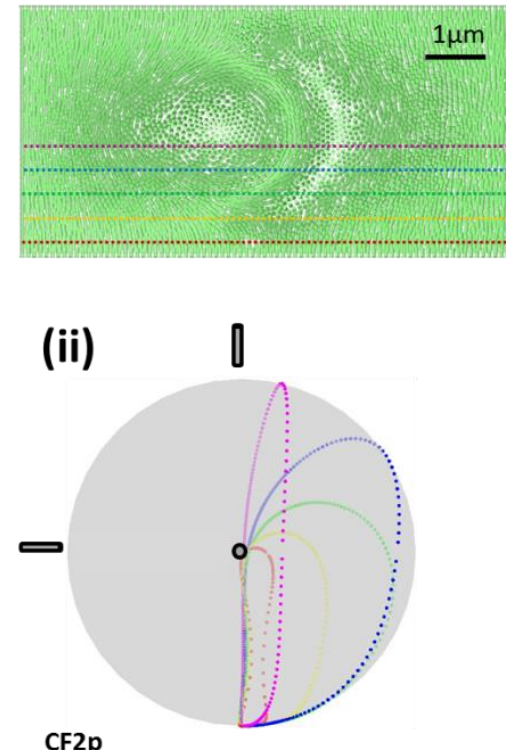

CF2p
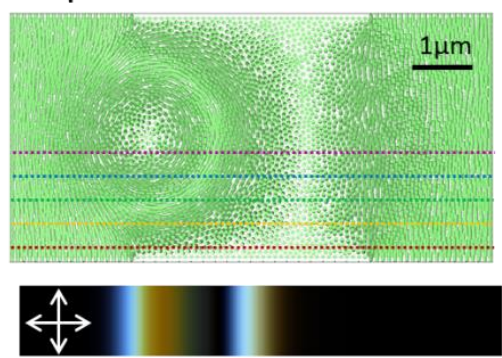

X (c)
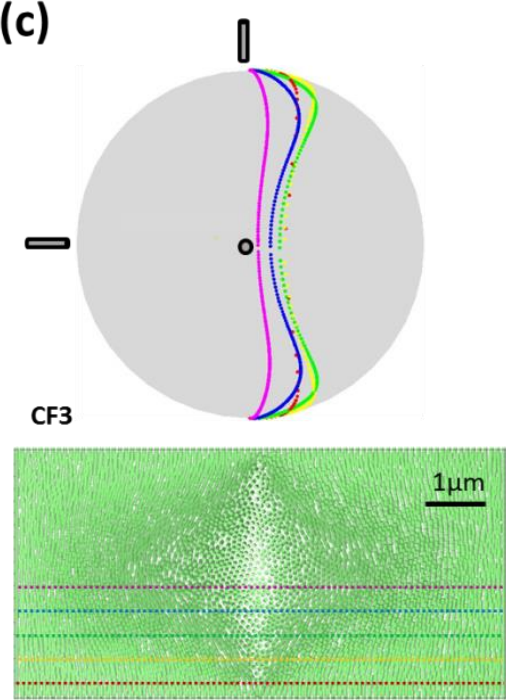

(iii)

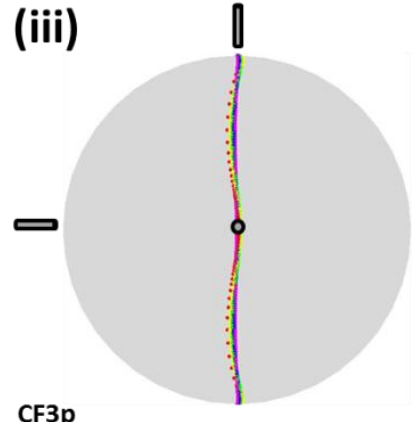

CF3p

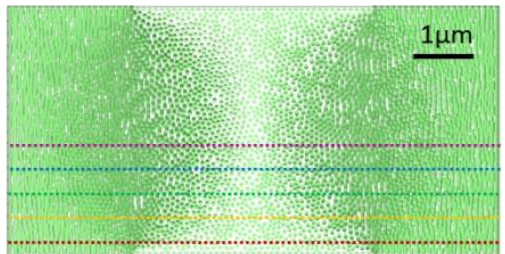

$\stackrel{\uparrow}{\gtrless}$

$\pi$ 


\section{WILEY-VCH}

The table of contents entry should be 50-60 words long, and the first phrase should be bold. The entry should be written in the present tense and impersonal style.

A novel mask-less photo-alignment technique with spatial control over polar and azimuthal anchoring is presented. A single layer of two-component alignment material is illuminated with two-beam UV interference to create periodic stripes with planar and homeotropic anchoring. This anchoring pattern helps to stabilize and align new chiral liquid crystal superstructures in confined geometries.

\section{Keyword}

Liquid crystals, photo-alignment, polar and azimuthal anchoring, cholesteric fingers

Inge Nys, Ke Chen, Jeroen Beeckman*, Kristiaan Neyts

Periodic Planar-Homeotropic Anchoring Realized by Photo-Alignment for Stabilization of Chiral Superstructures

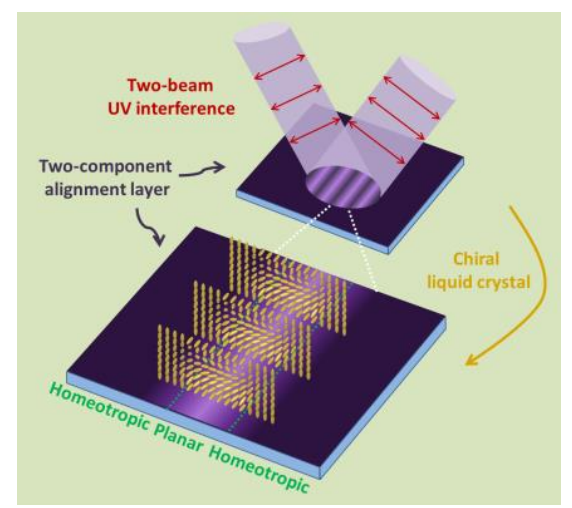




\section{WILEY-VCH}

Copyright WILEY-VCH Verlag GmbH \& Co. KGaA, 69469 Weinheim, Germany, 2016.

\section{Supporting Information}

\section{Periodic Planar-Homeotropic Anchoring Realized by Photo-Alignment for Stabilization of Chiral Superstructures}

Inge Nys, Ke Chen, Jeroen Beeckman*, Kristiaan Neyts

Verification of the proposed photo-alignment technique and comments about the stability:

Figure S1 shows the results for a cell filled with a mixture of NLC E7 and dichroic dye $(1 \mathrm{wt} \%)$. With the help of these experiments it can be confirmed that, in accordance with the theory, the alignment in the planar stripes is along the y-direction, parallel to the stripes.

Figure S2 shows the effect of an increased UV dose on the obtained surface alignment. The same interference illumination pattern was used (period $=9 \mu \mathrm{m})$ but the illumination time and power were increased to $3 \mathrm{~min}$ and $\approx 80 \mathrm{~mW}$. Only the edges of the illuminated area show the previously described stripes of planar and homeotropic anchoring while over-illumination in the central part leads to a uniform planar alignment. This demonstrates that the UV dose can control the transition between homeotropic and planar anchoring. Moreover, at the edge of the illuminated area approximately $1 \mu \mathrm{m}$ thick lines with planar anchoring are observed, which indicates that the polar anchoring strength in the planar regions is high enough to overcome the elastic energy of a rapid variation in the director.

The photo-stability and thermal stability of the proposed alignment material was not studied in detail but our experiments indicate that the induced alignment is well preserved for at least a couple of months when the cell is stored at room temperature in a dark environment. As demonstrated previously, to improve the stability of the system a thin layer of liquid crystal 


\section{WILEY-VCH}

monomer can be polymerized onto the alignment layer or the complete LC cell can be polymerized.

Comments about the molecular structure of the alignment layer:

The alignment layer consists of a mixture of homeotropic alignment material (SE 4811) and photo-alignment material (PAAD22). Multiple photo-alignment materials are available and a lot of them are based on azo-compounds but the working principles can be different.$^{[2]}$ The photo-alignment material PAAD 22 shows planar degenerate anchoring before UV illumination and planar alignment in one direction after linearly polarized UV illumination. The working principle is based on successive excitation and reorientation of the azo-based molecules whereby the chance for excitation depends on the orientation of the molecular absorption oscillator with respect to the direction of linear polarization of the light. This process of angular redistribution leads to photoinduced orientational ordering. ${ }^{[2]}$ The imidized polyimide SE 4811 has a relatively rigid backbone with alkyl side chains oriented perpendicular to the backbone. ${ }^{[3]}$ In normal working conditions, the alkyl side chains give rise to vertical alignment but strong UV illumination leads to bond breaking and cleavage of the aromatic and imide rings. At the same time, the UV light leads to a destruction of the backbone and surface anisotropy can be induced by polarized illumination. After long exposure with linearly polarized light this can lead to planar alignment perpendicular to the polarization of the UV light. ${ }^{[3]}$ We experimentally tested that for our illumination conditions no transition to planar alignment was observed in the pure SE 4811 mixture, but a cooperative effect with the azo-based photo-alignment molecules cannot be excluded in the alignment mixture. We did not make a detailed study of the molecular composition and behavior, but we believe that mainly the photo-reorientation of the PAAD 22 molecules is responsible for the observed planar alignment in the regions with high illumination intensity. 


\section{WILEY-VCH}

This effect can be further supported by cutting of the alkyl chains and introduction of surface anisotropy in the polyimide material.

Details of the simulation method:

The result of the Q-tensor simulation (on a triangular mesh) is interpolated onto a regular (x,z) grid and the azimuth and inclination of the director are calculated. The transmission for light propagating along the z-axis is calculated as a function of the x-coordinate, based on the Jones calculus, using the director values for the given x-coordinate. Relatively rapid spatial variations of the director along $\mathrm{x}$ are present and diffraction and refraction can play a role in our cells. Therefore the simulated transmission results can only give an approximation for the transmission between crossed polarizers, which is more reliable when the variation along $\mathrm{x}$ is smaller. To obtain a rough color image, we combined the transmissions calculated for three wavelengths in a color plot: $420 \mathrm{~nm}$ for blue, $520 \mathrm{~nm}$ for green and $620 \mathrm{~nm}$ for red. The refractive indices of the liquid crystal that are used in the simulations are no $=1.52$ and ne $=1.74$.

Figure S3 (obtained with the help of FE Q-tensor simulations) illustrates that when the pitch is decreased ( $p=8 \mu \mathrm{m}$ instead of $16 \mu \mathrm{m})$ the cholesteric twist axis of the CF1p finger is tilted further from the substrate normal and the structure better resembles the typical CF1 in homeotropic cells. This agrees with an increase in the maximum inclination angle $\alpha_{0}$, describing the deviation from the untwisted configuration, in homeotropically aligned cells. Figure S4 shows the CF3p configuration and its extension towards a configuration with $2 \pi$ twist. This structure contains two $\pi$ disclinations close to the top and bottom substrates and does not correspond to any observed director pattern. 


\section{WILEY-VCH}

Figure S1. Microscopy images with only one polarizer for a NLC cell with dichroic dye. A simplified director representation is also shown to indicate the homeotropic and planar stripes. These measurements confirm that the orientation in the planar region is along the y-axis (stripe direction).
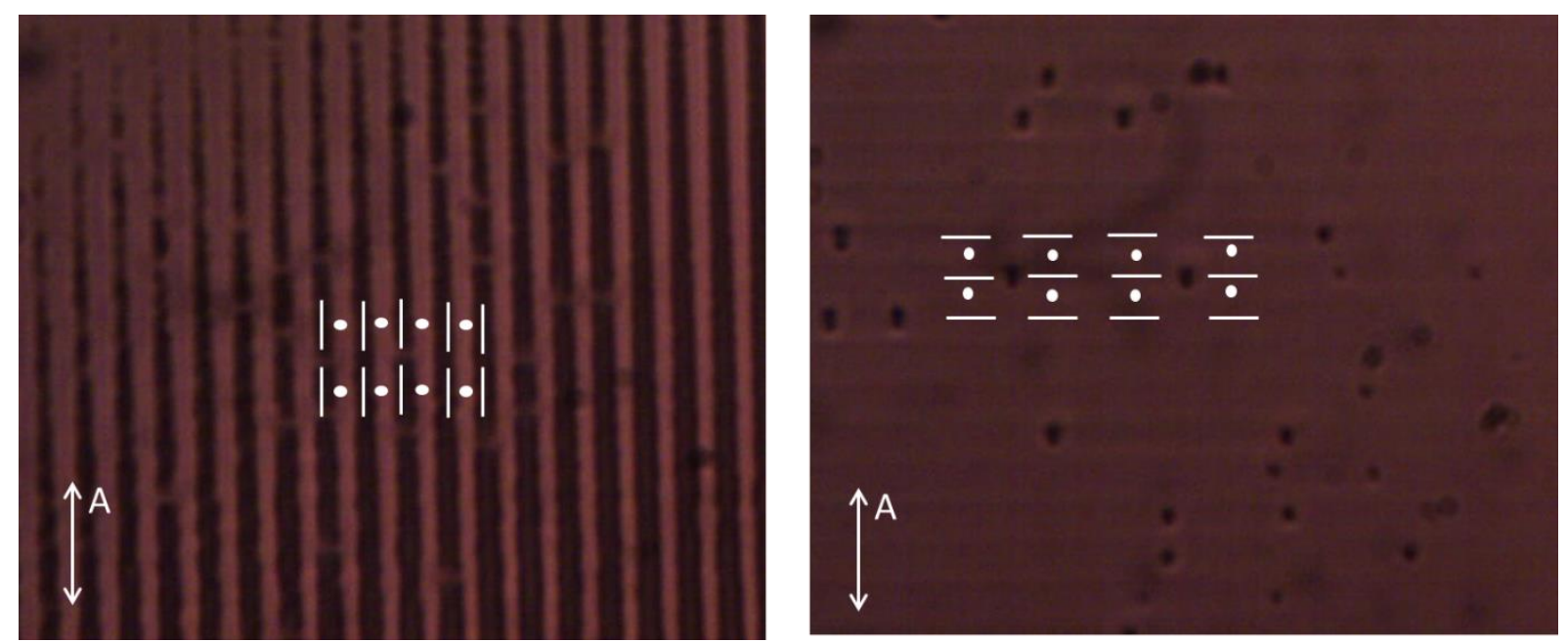

Figure S2. Polarizing microscopy images of a strongly illuminated cell filled with NLC (a smaller and larger magnification are shown). The cell was illuminated with the UV interference pattern (period $=9 \mu \mathrm{m}$ ) for 3 min with power $\approx 80 \mathrm{~mW}$ over an area of $\approx 0.4 \mathrm{~mm}^{2}$. The illuminated area is surrounded by a homeotropic region. The edges of the illuminated area show periodic stripes with planar and homeotropic alignment while the central area is over-illuminated and gives rise to uniform planar alignment along the y-axis.
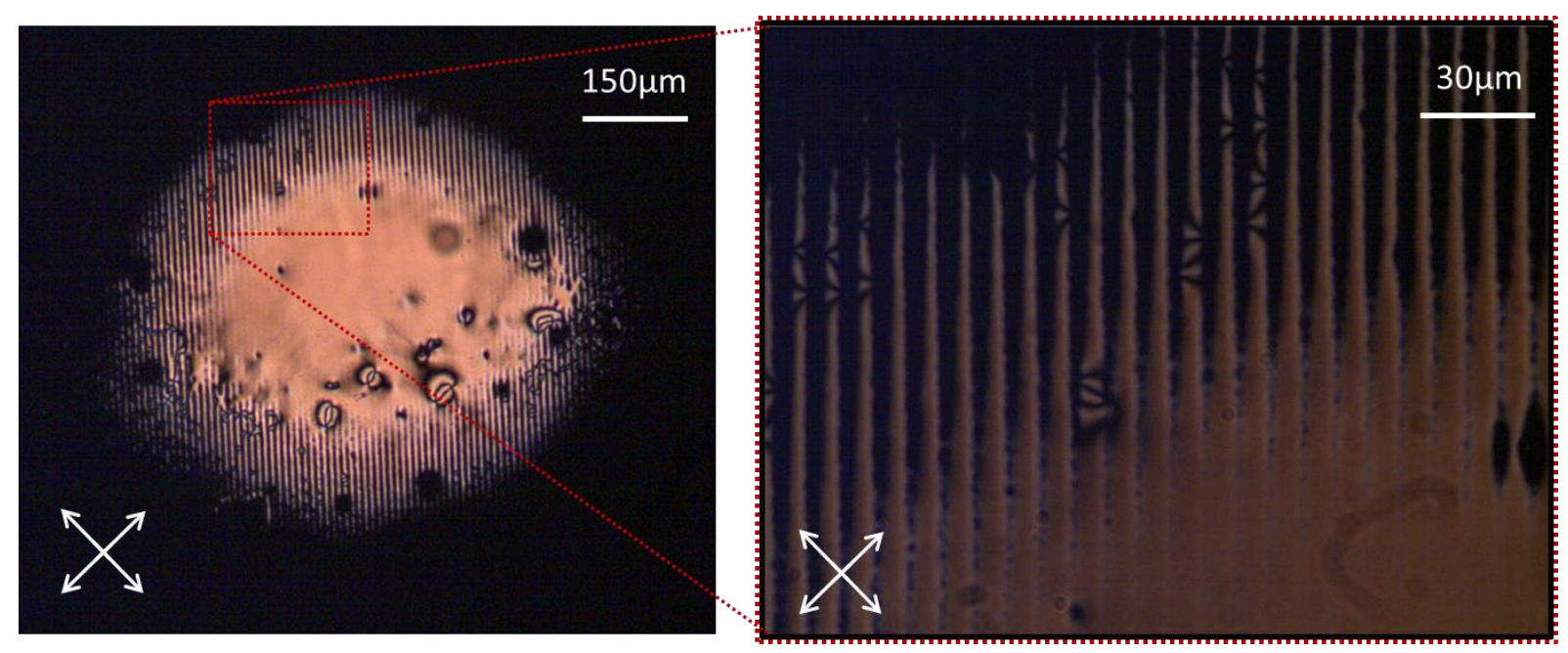


\section{WILEY-VCH}

Figure S3. Simulated projection of the end point of the director on the unit sphere (top), director configuration (middle) and transmission between crossed polarizers (bottom) for a CF1p cholesteric finger in a cell with periodic planar/homeotropic alignment. The pitch of the CLC was $8 \mu \mathrm{m}$ in a) and $16 \mu \mathrm{m}$ in b).
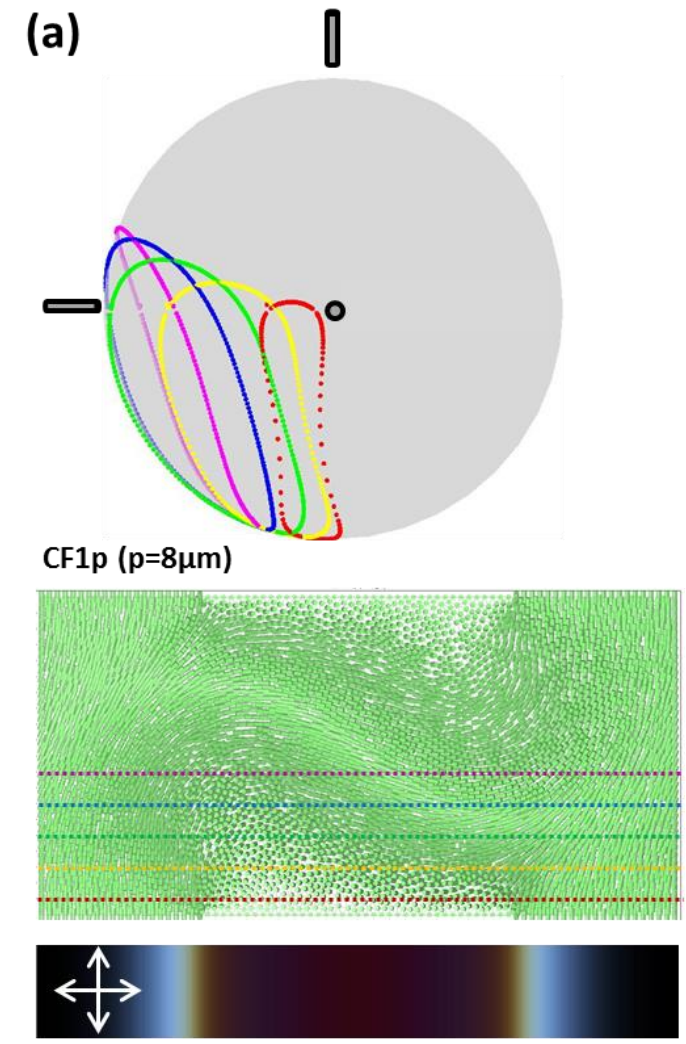

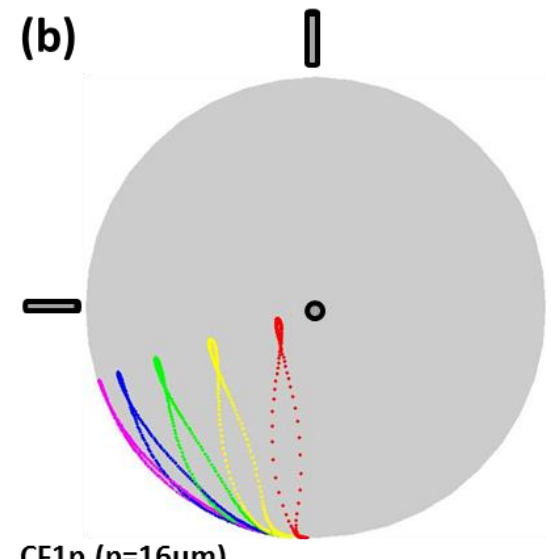

CF1p ( $p=16 \mu m)$
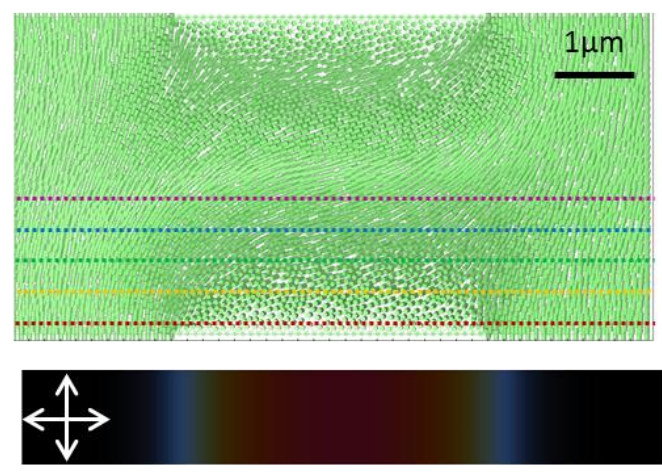

$\sum$ 


\section{WILEY-VCH}

Figure S4. Simulated projection of the director on the unit sphere (top), director configuration (middle) and transmission between crossed polarizers (bottom) for a CF3p with a $\pi$ twist (left) and another cholesteric finger with a $2 \pi$ twist (right) in a cell with periodic planar/homeotropic alignment. The pitch of the CLC was $16 \mu \mathrm{m}$.
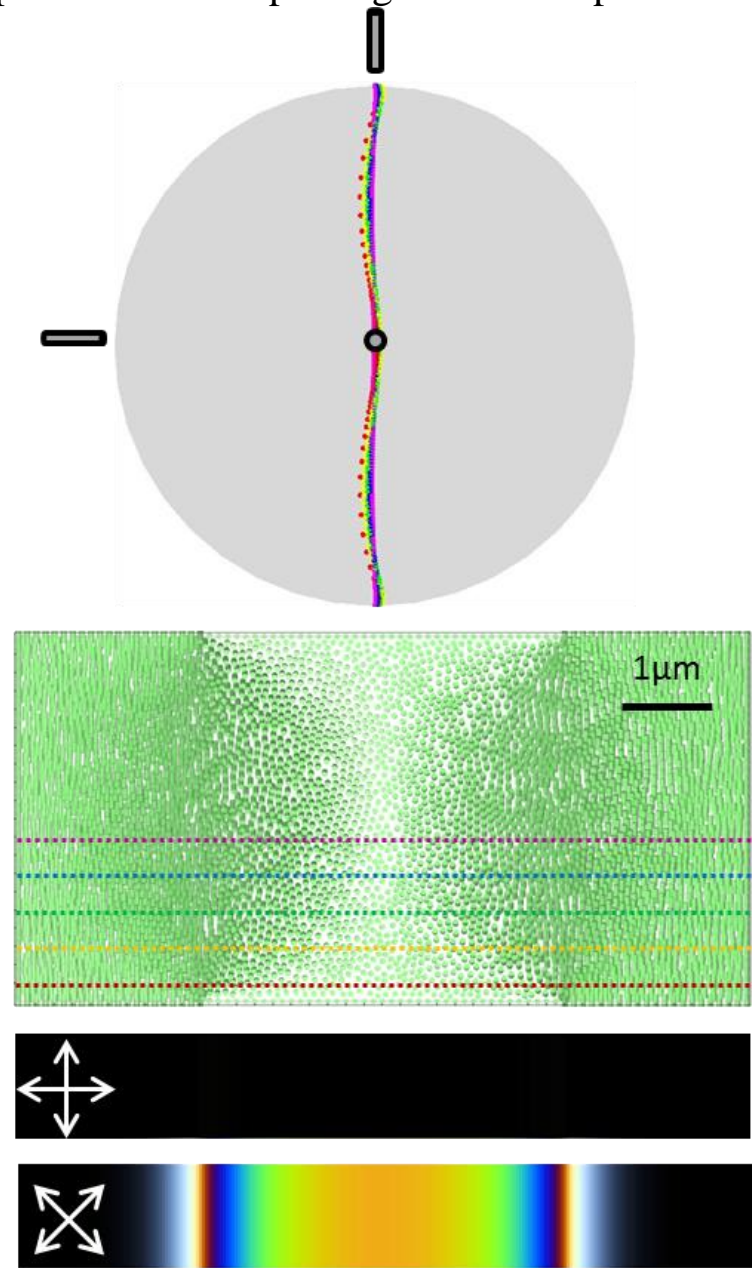
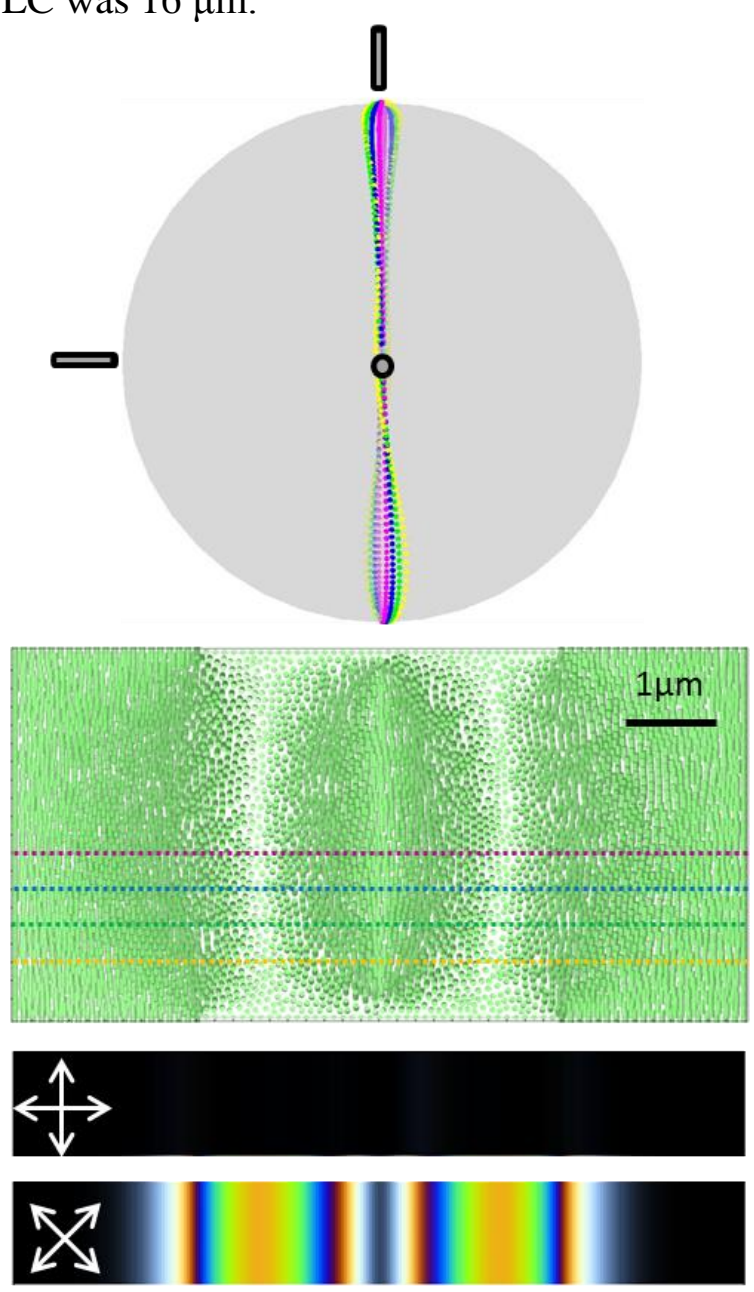

[1] O. Yaroshchuk, V. Kyrychenko, Du Tao, V. Chigrinov, H. S. Kwok, H. Hasebe,

H. Takatsu, Appl. Phys. Lett. 2009, 95, 021902.

[2] a) H. K. Bisoyi, Q. Li, Chem. Rev. 2016, 116, 15089; b) O. Yaroshchuk, Y. Reznikov, J.

Mater. Chem. 2012, 22, 286.

[3] R. A. Alla, G. Hegde, L. Komitov, Appl. Phys. Lett. 2013, 102, 233505. 